\title{
Corrigendum: Genome-wide genetic changes during modern breeding of maize
}

Yinping Jiao, Hainan Zhao, Longhui Ren, Weibin Song, Biao Zeng, Jinjie Guo, Baobao Wang, Zhipeng Liu, Jing Chen, Wei Li, Mei Zhang, Shaojun Xie \& Jingsheng Lai

Nat. Genet. 44, 812-815 (2012); published online 3 June 2012; corrected after print 27 August 2014

In the version of this article initially published, Figure 2 and related results were flawed because of errors in the analysis that incorrectly assigned the B73 reference genotype to non-overlapping SNP sites, resulting in SNPs being inappropriately combined. In addition, the authors have revised identity-by-descent (IBD) region identification using a 50-SNP sliding window with a step size of 5 SNPs and excluded regions with genetic distance of $\leq 0.05 \mathrm{cM}$. As a result of these changes, the authors have provided a corrected version of the paper to be appended to the original publication (the Online Methods, Figs. 1-4 and their legends, and Table 1 and its legend were revised). Minor revisions have also been made in the main text to reflect changes to calculations resulting from the above corrections (changes are made in paragraphs 2 and 3 of original page 812, paragraphs 1 and 2 of original page 813, and paragraphs 1-6 of original page 814). In addition, corrected versions of Supplementary Tables 2-6 and 11 and Supplementary Figures 1, 2, 5 and 6, and two new supplementary figures and one new supplementary table have been added. The main conclusions of the paper were not affected by the corrections.
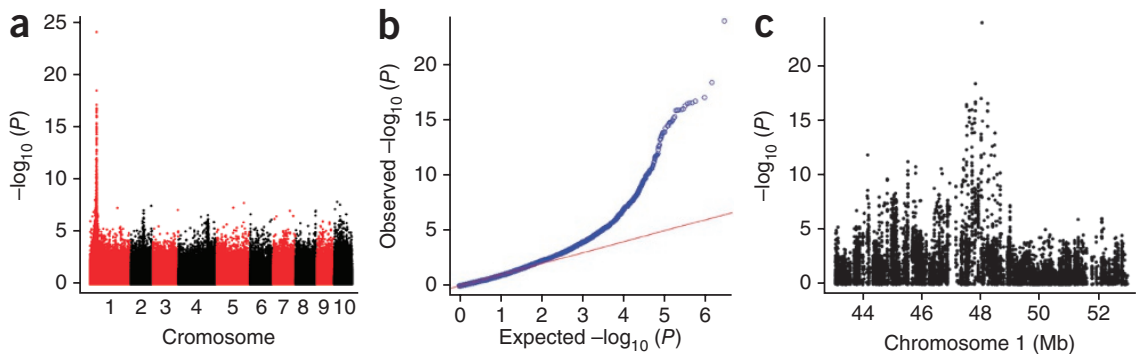

Figure 1 GWAS results for cob color. (a) Manhattan plot. (b) Quantile-quantile plot. (c) Regional Manhattan plot of $5 \mathrm{Mb}$ on either side of the peak SNP.

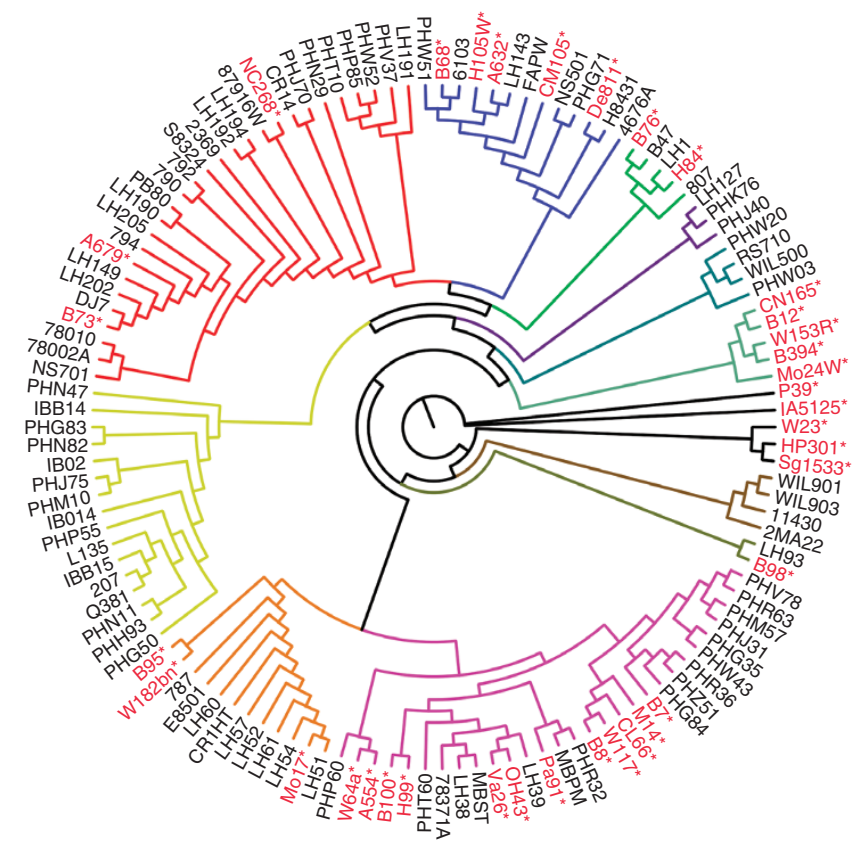

Figure 2 Neighbor-joining tree of the 126 US maize inbred lines. Lines in the public US group are shown in red followed by an asterisk. Ex-PVP lines are shown in black.

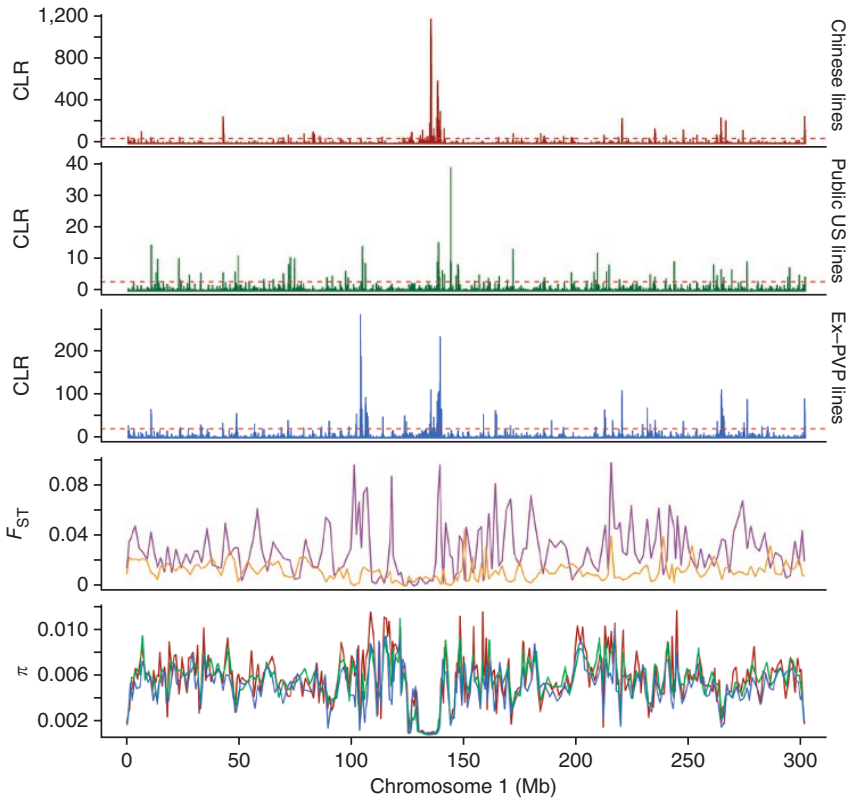

Figure 3 CLR and genetic diversity of chromosome 1 in public US, Ex-PVP and elite Chinese maize groups. Green lines, public US group; blue lines, Ex-PVP group; red lines, elite Chinese group; orange lines, $F_{\mathrm{ST}}$ of the Chinese and US (both public and Ex-PVP) maize groups; purple lines, $F_{\mathrm{ST}}$ of the public US group and Ex-PVP groups. 


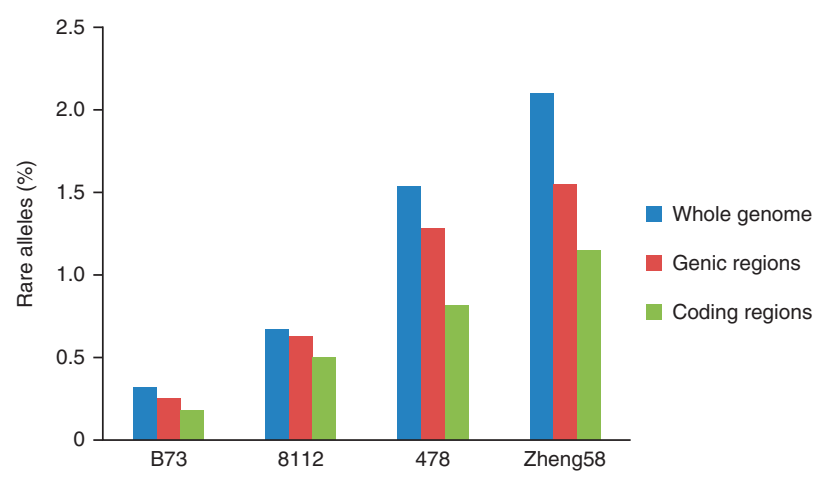

Figure 4 The percentage of rare alleles in four related inbred lines. Allele frequencies were calculated in the 278 Chinese and US lines. Rare alleles are defined as those present in $\leq 5 \%$ of the sequenced lines. B73 is known to be the ancestral line of 8112,478 and Zheng58. All share the lowa Stiff Stalk background.
Table 1 Mutation rates of base substitutions, short indels and copy number variations

\begin{tabular}{lccc}
\hline & $5003 \rightarrow 478^{a}$ & $8112 \rightarrow 478^{a}$ & $478 \rightarrow Z 58^{b}$ \\
\hline $\begin{array}{c}\text { Number of years since } \\
\text { breeding }\end{array}$ & 26 & 26 & 24 \\
$\begin{array}{c}\text { Number of sites surveyed } \\
\quad \text { (all three generation with } \\
\text { sequencing depth of } \geq 5)\end{array}$ & $170,469,103$ & $317,986,593$ & $488,466,696$ \\
$\begin{array}{c}\text { Number of single-base } \\
\text { mutations }\end{array}$ & 179 & 164 & 441 \\
$\begin{array}{c}\text { Number of single-base } \\
\text { mutations in genic regions }\end{array}$ & 20 & 13 & 48 \\
$\begin{array}{c}\text { Number of single-base } \\
\text { mutations in coding regions }\end{array}$ & 6 & 9 & 13 \\
Number of nonsynonymous & 5 & 6 & 9
\end{tabular}

single-base mutations

Single-base substitution rate (per site per year)

Number of 1- to 10-bp indels

Mutation rate of 1 - to $10-\mathrm{bp}$ indels (per site per year)

Number of genes surveyed

Number of CNV genes

CNV mutation rate

(per gene per year)

$2.02 \times 10^{-8} \quad 9.92 \times 10^{-9} \quad 1.88 \times 10^{-8}$

$18 \quad 18 \quad 58$

$2.03 \times 10^{-9} \quad 1.09 \times 10^{-9} \quad 2.47 \times 10^{-9}$

$5,409 \quad 8,603 \quad 14,012$

$103 \quad 113 \quad 305$

$3.66 \times 10^{-4} \quad 2.53 \times 10^{-4} \quad 4.19 \times 10^{-4}$

anly the mutations transferred from 478 to $\mathrm{Z} 58$ were considered. ${ }^{\mathrm{b}}$ Only the mutation sites showing no polymorphism in comparison of 8112 and 478 or 5003 and 478 were considered.

\section{Corrigendum: A copy number variation morbidity map of developmental delay}

Gregory M Cooper, Bradley P Coe, Santhosh Girirajan, Jill A Rosenfeld, Tiffany H Vu, Carl Baker, Charles Williams, Heather Stalker, Rizwan Hamid, Vickie Hannig, Hoda Abdel-Hamid, Patricia Bader, Elizabeth McCracken, Dmitriy Niyazov, Kathleen Leppig, Heidi Thiese, Marybeth Hummel, Nora Alexander, Jerome Gorski, Jennifer Kussmann, Vandana Shashi, Krys Johnson, Catherine Rehder, Blake C Ballif, Lisa G Shaffer \& Evan E Eichler Nat. Genet. 43, 838-846 (2011); published online 14 August 2011; corrected after print 27 August 2014

In the version of this article initially published, in Table 1 and its associated text, there was a calculation error in which the relative sizes of the case and control populations were set to be equal; because the size of the case population $(15,767)$ was nearly double that of the control population $(8,329)$, this resulted in erroneously inflated penetrance estimates. A simple definition of penetrance is used that is often applied in medical genetics-namely, the proportion of observed mutation carriers that are affected-to provide a metric that would be useful to clinical geneticists in a setting in which disease is heavily enriched, for example, in diagnosing children with developmental delay. That formulation is biased upwards with respect to population-level penetrance. Thus, in this corrigendum, an estimate more appropriate for population-level inference is provided assuming a general disease prevalence of 5.3\% (Am. J. Hum. Genet. 42, 677-693, 1988) along with the more familiar odds ratio (OR) estimate. Importantly, all of these measures of penetrance are intrinsically limited by sampling error and imprecision in defining disease prevalence. We note that the mutation carrier counts, $P$ values and other results in the original version of Table 1 are correct, and the key results and conclusions of the paper are unaffected. The error has been corrected in the HTML and PDF versions of the article.

\section{Corrigendum: Genome-wide association analyses of esophageal squamous cell carcinoma in Chinese identify multiple susceptibility loci and gene- environment interactions}

Chen Wu, Peter Kraft, Kan Zhai, Jiang Chang, Zhaoming Wang, Yun Li, Zhibin Hu, Zhonghu He, Weihua Jia, Christian C Abnet, Liming Liang, Nan Hu, Xiaoping Miao, Yifeng Zhou, Zhihua Liu, Qimin Zhan, Yu Liu, Yan Qiao, Yuling Zhou, Guangfu Jin, Chuanhai Guo, Changdong Lu, Haijun Yang, Jianhua Fu, Dianke Yu, Neal D Freedman, Ti Ding, Wen Tan, Alisa M Goldstein, Tangchun Wu, Hongbing Shen, Yang Ke, Yixin Zeng, Stephen J Chanock, Philip R Taylor \& Dongxin Lin Nat. Genet. 44, 1090-1097 (2012); published online 9 September 2012; corrected after print 27 August 2014 